\title{
Aged garlic extract ameliorates fatty liver and insulin resistance and improves the gut microbiota profile in a mouse model of insulin resistance
}

\author{
TOSHIO MAEDA ${ }^{1}$, SATOMI MIKI ${ }^{2}$, NAOAKI MORIHARA ${ }^{2}$ and YOSHIYUKI KAGAWA $^{1}$ \\ ${ }^{1}$ Department of Clinical Pharmaceutics and Pharmacy Practice, \\ Graduate School of Pharmaceutical Sciences, University of Shizuoka, Shizuoka 422-8526; \\ ${ }^{2}$ Central Research Institute, Wakunaga Pharmaceutical Co., Ltd., Akitakata, Hiroshima 739-1195, Japan
}

Received April 22, 2019; Accepted May 30, 2019

DOI: $10.3892 /$ etm.2019.7636

\begin{abstract}
Aged garlic extract (AGE) produced by the aging process has various beneficial pharmacological effects. In this study, the effects of AGE on fatty liver, insulin resistance and intestinal microbiota were compared between ddY-H mice, an insulin resistance mouse, and ddY-L mice, normal mice. Mice were fed an AGE-supplemented diet (4\% w/w) for 7 weeks. The administration of AGE had no effect on the body weight and dietary intake of both types of mice. In the ddY-H mice, the serum levels of glucose and insulin were increased and glucose tolerance was impaired; however, the administration of AGE ameliorated these abnormal conditions. AGE did not have these effects in ddY-L mice. Triglyceride (TG) accumulation in the liver and fat absorption from the digestive tract were increased in the ddY-H mice; however, the administration of AGE reduced this increase. On the other hand, AGE exerted no such effects in the ddY-L mice. In addition, the gut microbiota has been shown to be closely associated with obesity, diabetes, dyslipidemia and non-alcoholic fatty liver disease in human and animal models. The bacterial composition of the gut microbiota in the feces of the ddY-H mice did not differ from that of the ddY-L mice at 5 weeks of age; however, it was altered in the mice at 9 and 12 weeks of age even when the mice were fed a standard diet. In the ddY-H mice, the relative presence of Lactobacillales was increased, while that of Bifidobacterium, Clostridium cluster XVIII and Prevotella was decreased. The alteration of the bacterial composition in the ddY-H mice was reversed by the administration of AGE; however, this effect of AGE was not observed in the ddY-L mice. On the whole, the
\end{abstract}

Correspondence to: Dr Toshio Maeda, Department of Clinical Pharmaceutics and Pharmacy Practice, Graduate School of Pharmaceutical Sciences, University of Shizuoka, 52-1 Yada, Suruga-ku, Shizuoka 422-8526, Japan

E-mail: maeda@u-shizuoka-ken.ac.jp

Key words: aged garlic extract, fatty liver, intestinal fat absorption, insulin resistance, gut microbiota findings of this study indicate that AGE improves abnormal fat accumulation and insulin resistance, and also alters the intestinal flora in ddY-H mice, suggesting the possibility that these effects of AGE may be related.

\section{Introduction}

Non-alcoholic fatty liver disease (NAFLD) is one of the most prevalent liver diseases worldwide. NAFLD involves the abnormal accumulation of triglycerides (TG) in hepatocytes (1). NAFLD progresses through non-alcoholic steatohepatitis (NASH) to cirrhosis and hepatocellular carcinoma. NAFLD is considered to be a hepatic manifestation of metabolic syndrome (2-4) and is closely linked to hepatic insulin resistance and abnormal glucose metabolism $(5,6)$. Epidemiological studies have demonstrated that NAFLD is associated with the development of type II diabetes (T2D) and that the improvement of NAFLD will result in a decreased risk of developing T2D (7). Therefore, NAFLD and T2D appear to be closely associated. A recent study demonstrated that the activation of transcription factor 3 is a key molecule linking NAFLD to impaired glucose homeostasis in Zucker diabetic fatty rats and in patients with NAFLD (8).

In recent years, a number of studies have revealed that the gut microbiota can be a potential contributor to the risk of obesity (9), diabetes (10), dyslipidemia (11) and NAFLD $(12,13)$ in humans and animal models. The perturbation of the gut microbiota composition has been observed in patients suffering from NAFLD (14). The quantities of the gut microbiota differ significantly between patients with T2D and healthy individuals (15). It has been demonstrated that a high-fat diet, which induces NAFLD and insulin resistance, can cause imbalances in the gut microbiota composition of mice and that germ-free animals are protected from the deleterious effect of a high-fat diet $(16,17)$. In rats fed a high-fat diet, an imbalance of the gut microbiota has been shown to be markedly associated with the progression of NAFLD (18). Probiotics (Lactobacillus rhamnosus, Lactobacillus acidophilus and Bifidobacterium bifidum) have been shown to modulate the gut microbiota and improve insulin sensitivity in mice fed a high-fat diet (16). Therefore, the manipulation of the gut microbiome by 
probiotics or prebiotics is an important means with which to prevent and treat NAFLD and insulin resistance in patients, as well as in rodent models $(14,16,19)$.

Garlic (Allium sativum L.) has been known as a medicinal food due to its various biological effects, such as antioxidant (20), anti-inflammatory (21), antimicrobial (22), antithrombotic (23) and antitumor effects (24). Aged garlic extract (AGE) is prepared through extraction and aging in a water-ethanol mixture for $>10$ months at room temperature (25). By this process, the majority of the molecules responsible for the harsh and irritating flavors which are typical of fresh raw garlic (i.e., allicin) are converted into more stable compounds, such as $S$-allylcysteine (SAC) and $S$-allylmercaptocysteine (SAMC), which are recognized as potent antioxidants (26). As regards the anti-diabetic and anti-obesity effects, some studies have demonstrated the beneficial effects of AGE in improving lipid profiles and insulin sensitivity in animals and humans $(25,27)$. AGE and its individual components have been shown to exert a protective effect against experimental hepatic injuries (28-30). It has been reported that SAC improves NAFLD in rats with T2D via the regulation of hepatic lipogenesis and glucose metabolism (31). On the other hand, some evidence for the beneficial effects of AGE on the gut microbiota has been reported in animals and humans. A previous study demonstrated that 3 months of AGE supplementation in humans improved the gut microbiota, as evidenced by greater microbial richness and diversity with a marked increase in Lactobacillus and Clostridium species (32).

Numerous animal models of NAFLD and diabetes, which are classified into genetic models and nutritional models, have been reported to date. As nutritional models, rodents fed a high-fat diet (33) have been reported. $\mathrm{db} / \mathrm{db}$ mice (34), ob/ob mice (35), KK-A $\mathrm{A}^{\mathrm{y}}$ mice (36) and the others are known as genetic models. However, in these models, apart from being fed a high-fat diet (16), an association of a perturbation of the gut microbiota with fatty liver and metabolic abnormalities, such as obesity, insulin resistance, dyslipidemia and altered adipokine profiles, as observed in patients with NAFLD has not been shown. We previously succeeded in isolating and breeding spontaneous insulin-resistant mice and healthy control mice, termed ddY-H and ddY-L mice, respectively (37). In ddY-H mice, hepatic steatosis with obesity, insulin resistance, hyperglycemia and altered adipokine profiles are observed even when the mice are fed a standard diet containing $13 \%$ energy from fat and $61 \%$ carbohydrates, but not so in ddY-L mice (37-40). The prevailing hypothesis is that hepatic steatosis develops as a result of the increased absorption of fat from the intestines and the increased uptake of fatty acids into hepatic cells via the increased expression of peroxisome proliferator-activated receptor $\gamma$ (PPAR $\gamma$ ) (39). The age-dependent onset of insulin resistance with hyperinsulinemia in ddY-H mice may be attributed to the decreases in the mRNA expression and proteins of insulin receptor, insulin receptor substrate (IRS)-1 and IRS-2 in adipose tissue (40).

The effects of AGE on abnormal fatty acid metabolism and insulin resistance related to the gut microbiota in an animal genetic model with NAFLD have not yet been studied, at least to the best of our knowledge. Thus, in the present study, the effects of AGE on fatty liver, insulin resistance and the gut microbiota, and their possible association was investigated by feeding an AGE-supplemented diet to ddY-H mice, an animal model with NAFLD and insulin resistance.

\section{Materials and methods}

Diet supplemented with AGE. Standard chow pellets (CLEA Rodent Diet CE-2, CLEA Japan, Inc.) supplemented with or without $4 \%(\mathrm{w} / \mathrm{w})$ AGE were provided by Wakunaga Pharmaceutical Co., Ltd.

Experimental design. A total of 16 male ddY-H mice and 16 male ddY-L mice (both 6 weeks old) derived from our own colony were used. The mice were divided into 2 groups, namely the AGE group and control group, with 8 mice in each group. The body weights of the ddY-H mice and ddY-L mice were $39.78 \pm 1.82$ and $31.40 \pm 2.61$, respectively. They were housed at $22^{\circ} \mathrm{C}$ to $24^{\circ} \mathrm{C}$ with 50 to $60 \%$ humidity under artificial lighting conditions with a 12-h light/dark cycle and maintained with free access to the diet supplemented with or without $4 \%$ (w/w) AGE and water ad libitum from 6 to 13 weeks of age. At 13 weeks of age, fecal sample collection for microbiota analysis, and a glucose tolerance test, the measurement of TG absorption from the intestines and the evaluation of various metabolic parameters were carried out. Animal care and experiments were performed in accordance with the guidelines for the care and use of laboratory animals of the University of Shizuoka, and animal experiments were reviewed and approved by the Animal and Ethics Review Committee of University of Shizuoka (no. 136004).

Treatment with antibacterial drugs. The ddY-H and ddY-L mice from our colony were fed standard chow pellets (CLEA Rodent Diet CE-2, CLEA Japan, Inc.) as described above. Anti-bacterial drugs (10 mg/kg ampicillin, $10 \mathrm{mg} / \mathrm{kg}$ erythromycin, $5 \mathrm{mg} / \mathrm{kg}$ metronidazole and $10 \mathrm{mg} / \mathrm{kg}$ neomycin) were orally administered to the mice from 6 to 13 weeks of age as previously described (41) prior to the glucose tolerance test and the measurement of hepatic TG levels. The anti-bacterial drugs were purchased from Tokyo Chemical Industry Co., Ltd.

Body mass index (BMI). BMI was calculated according to the following formula: Body weight $(\mathrm{g}) /$ body length ${ }^{2}\left(\mathrm{~cm}^{2}\right)$, as previously described (38).

Fecal sample collection and microbiota analysis. The mice were moved to new cages at 09:00 and fresh fecal samples were collected for $30 \mathrm{~min}$. DNA extraction, polymerase chain reaction and terminal restriction fragment length polymorphism (T-RFLP) analysis for the composition of the microbiota were carried out at Techno Suruga Laboratory Co., Ltd. As previously described by Nagashima et al $(42,43)$. Briefly, the fecal sample was suspended in a solution containing $100 \mathrm{mM}$ Tris- $\mathrm{HCl}$ (pH 9.0) and $40 \mathrm{mM}$ EDTA after washing 3 times with sterile distilled water, then beaten in the presence of glass beads using a mini-bead beater (BioSpec Products). Thereafter, DNA was extracted from the bead-treated suspension using benzyl chloride and was purified using a GFX PCR DNA and Gel Band Purification kit (Amersham Biosciences). PCR of DNA was performed with a model thermal cycler (Applied Biosystems) in a reaction mixture containing HotStarTaq 
DNA polymerase (Qiagen) and primers, 5' HEX-labeled $516 \mathrm{f}$ and 1510r. The products were digested with $\mathrm{B} s / \mathrm{I}$ for $1 \mathrm{~h}$ and then fractionated using an ABI PRISM 310 Genetic Analyzer (Applied Biosystems). The length and peak area of terminal restriction fragments (T-RFs) in the electropherogra ms were determined using genotype software GeneMapper (Applied Biosystems). The T-RFs were divided into 29 operational taxonomic units (OTUs). The OTUs were quantified as the percentage of individual OTU area per the total OTU area under the curve (AUC). In addition, the presence of Bifidobacterium was evaluated by anaerobical cultivation on selective BS agar plate and real-time PCR. Briefly, the cultivation was carried out as follows: $0.2 \mathrm{~g}$ feces were washed with saline and then vigorously stirred in $10 \mathrm{ml}$ saline. Following centrifugation at $1,000 \mathrm{x} \mathrm{g}$ for $20 \mathrm{~min}$ at $4^{\circ} \mathrm{C}$, the supernatant was applied on a BS agar plate (Nissui Pharmaceutical Co., Ltd.) and the plate was anaerobically incubated at $37^{\circ} \mathrm{C}$ for 4 days. After the incubation, each plate was examined for bacterial colonies. For quantitative PCR, DNA was extracted from feces by using MORA-EXTRACT (Kyokuto Pharmaceutical Industrial Co., Ltd,). Quantitative PCR with the specific primers and SYBR ${ }^{\circledR}$ Premix ExTaq GC (Takara Bio Inc.) was carried out using an Applied Biosystems 7500 Real-Time PCR System (Life Technologies). The thernocycling conditions were as follows: Initial denaturation at $95^{\circ} \mathrm{C}$ for $30 \mathrm{sec}$ followed by 40 cycles of denaturation at $95^{\circ} \mathrm{C}$ for $10 \mathrm{sec}$, annealing at $58^{\circ} \mathrm{C}$ for $30 \mathrm{sec}$ and extension at $72^{\circ} \mathrm{C}$ for $30 \mathrm{sec}$. The final extension was conducted at $72^{\circ} \mathrm{C}$ for $1 \mathrm{~min}$. The primers used were as follows: The forward primer of 5'-TCGCGTCCGGTGTGAAAG-3' and the reverse primer of 5'-CCACATCCAGCATCCAC-3' for Bifidobacterium as a target gene and the forward primer of 5'-CCTACGGGAGGCAGCAG and the reverse primer of 5'CCACATCCAGCATCCAC-3' for 16S rDNA as control gene. Relative gene expression was quantified by using the $2^{-\Delta \Delta \mathrm{Cq}}$ method (44).

Measurement of serum glucose and insulin levels. Blood was obtained following a 12 h-fast (21:00-09:00). The serum glucose, insulin and leptin levels were determined using the Glucose CII Test Wako (Wako Pure Chemical Industries, Ltd.) and the Mouse Insulin ELISA kit (Morinaga Institute of Biological Sciences, Inc.), respectively. The insulin sensitivity index and insulin resistance index were assessed by the glucose and insulin levels in the following manner: Glucose (mg/dl)/insulin (ng/dl) and glucose (mg/dl) x insulin (ng/ml)/100, respectively (37).

Glucose tolerance test. The mice were fasted for $4 \mathrm{~h}$ (09:00-13:00) and were orally administered glucose (3 g/ kg). A total of $20 \mu \mathrm{l}$ of blood was collected from the caudal vein of each mouse after 0, 30, 60 and $120 \mathrm{~min}$. Serum glucose levels were measured, and the AUC of the time-glucose concentration was calculated (37).

Measurement of TG contents in serum and liver. All mice were sacrificed to obtain serum and livers at 13:00 following a 4-h fast. Serum TG levels were measured using the Triglyceride E-Test (Wako Pure Chemical Industries, Ltd.). For the determination of hepatic TG levels, TG in $0.2 \mathrm{~g}$ liver was extracted by $4 \mathrm{ml}$ of 2-propanol and the aliquot of the filtrate was

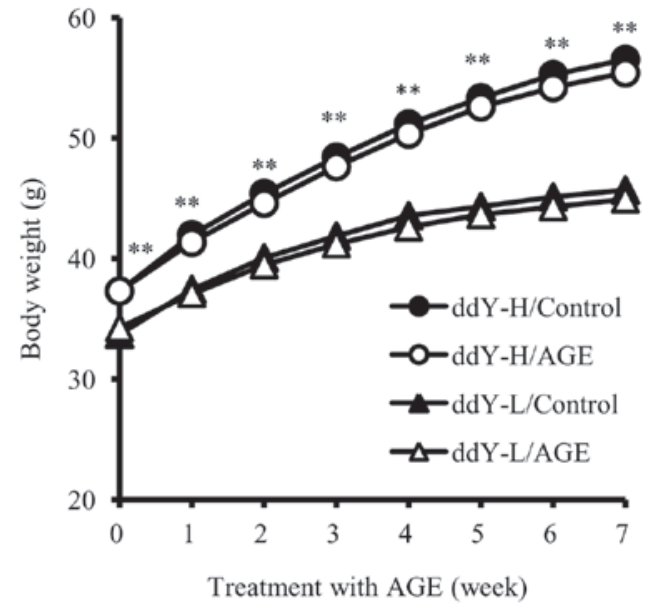

Figure 1. Effects of AGE on body weight in ddY-H mice and ddY-L mice. Each value represents the means $\pm \mathrm{SD}$ for 8 mice. ${ }^{* *} \mathrm{P}<0.01 \mathrm{vs}$. ddY-L/Control. AGE, aged garlic extract.

measured using the Triglyceride E-Test (Wako Pure Chemical Industries, Ltd.) (39).

Measurement of fat absorption from the intestines. To evaluate fat absorption from the intestines, olive oil $(10 \mathrm{ml} / \mathrm{kg}$ body weight) was orally administered to the mice. After 1, 2, 4 and $6 \mathrm{~h}, 20 \mu \mathrm{l}$ of blood were collected from the caudal vein of each mouse and serum TG concentrations were measured as described above. The area of increased TG concentration after olive oil loading in the serum TG concentration-time curve was calculated for the evaluation of TG absorption, as previously described (39).

Statistical analysis. All data are expressed as the means \pm SD. One-way analysis of variance (ANOVA) was used to compare the means among different groups, and Tukey's test was used in the post hoc multiple comparison. A value of $\mathrm{P}<0.05$ was considered to indicate a statistically significant difference.

\section{Results}

Effect of AGE on dietary intake, body weight and BMI. ddY-H mice exhibit the age-dependent onset of metabolic abnormalities, such as obesity, insulin resistance, hyperglycemia and an altered adipokine profile accompanied by a fatty liver when the mice are fed a standard diet (37-40). These changes are similar to those observed in patients with NAFLD. In this study, in order to examine the effects of AGE on insulin resistance and fatty liver in ddY-H mice, the mice were fed a standard diet supplemented with or without $4 \%$ (w/w) AGE for 7 weeks. The intake of the control diet in the ddY-H mice during the experimental period was $15 \%$ greater than that in the ddY-L mice during the experimental period. However, AGE supplementation had no effect on dietary intake in either the ddY-H or ddY-L mice throughout the experimental period (Table I). The body weight of the ddY-H mice was significantly greater than that of the ddY-L mice at the starting time (6 weeks of age) and throughout the experimental period. However, AGE supplementation did not influence the body weight of either of the types of mice (Fig. 1). At 13 weeks of age, the BMI 
Table I. Effect of AGE on dietary intake in ddY-H mice and ddY-L mice.

\begin{tabular}{lrrrr}
\hline Parameters & ddY-H/Control & ddY-H/AGE & ddY-L/Control & ddY-L/AGE \\
\hline Dietary intake & $316.54 \pm 30.41^{\text {a }}$ & $316.13 \pm 30.70$ & $276.82 \pm 29.45$ & $274.83 \pm 28.71$ \\
BMI & $0.46 \pm 0.056^{\mathrm{a}}$ & $0.45 \pm 0.051$ & $0.37 \pm 0.036$ & $0.036 \pm 0.029$ \\
\hline
\end{tabular}

Mice were fed a standard diet with and without $4 \%$ AGE for 7 weeks. Dietary intake of each mouse (g/49 days) during the experimental period was measured. BMI of each mouse after feeding for 7 weeks was measured as described in the Materials and methods. Each value represents the means \pm SD of 8 mice. ${ }^{a}<<0.01 v s$. ddY-L/Control. AGE, aged garlic extract.

Table II. Effect of AGE on serum levels of glucose, triglyceride and insulin in ddY-H and ddY-L mice.

\begin{tabular}{lccrr}
\hline Parameters & ddY-H/Control & ddY-H/AGE & ddY-L/Control & ddY-L/AGE \\
\hline Glucose (mg/dl) & $167 \pm 25.6^{\mathrm{a}}$ & $146 \pm 23.3^{\mathrm{b}}$ & $116 \pm 8.6$ & $124 \pm 10.5$ \\
Triglycerides (mg/dl) & $211 \pm 20.6^{\mathrm{a}}$ & $208 \pm 20.6^{\mathrm{b}}$ & $155 \pm 11.3$ & $159 \pm 20.6$ \\
Insulin $(\mathrm{ng} / \mathrm{ml})$ & $11.8 \pm 3.93^{\mathrm{a}}$ & $6.6 \pm 2.41 \mathrm{~b}$ & $2.3 \pm 1.14$ & $2.5 \pm 1.41$ \\
Insulin sensitivity index & $14.2 \pm 2.23^{\mathrm{a}}$ & $22.1 \pm 2.56^{\mathrm{b}}$ & $50.4 \pm 4.56$ & $49.6 \pm 6.78$ \\
Insulin resistance index & $19.7 \pm 2.31^{\mathrm{a}}$ & $9.6 \pm 1.96^{\mathrm{b}}$ & $2.7 \pm 1.07$ & $3.1 \pm 1.56$ \\
\hline
\end{tabular}

Mice were fed the standard diet with and without $4 \%$ AGE for 7 weeks and blood was withdrawn after 12-h fast. Each value represents the means \pm SD of 8 mice. ${ }^{a} \mathrm{P}<0.01$ vs. ddY-L/Control; ${ }^{b} \mathrm{P}<0.01$ vs. ddY-H/Control. AGE, aged garlic extract.
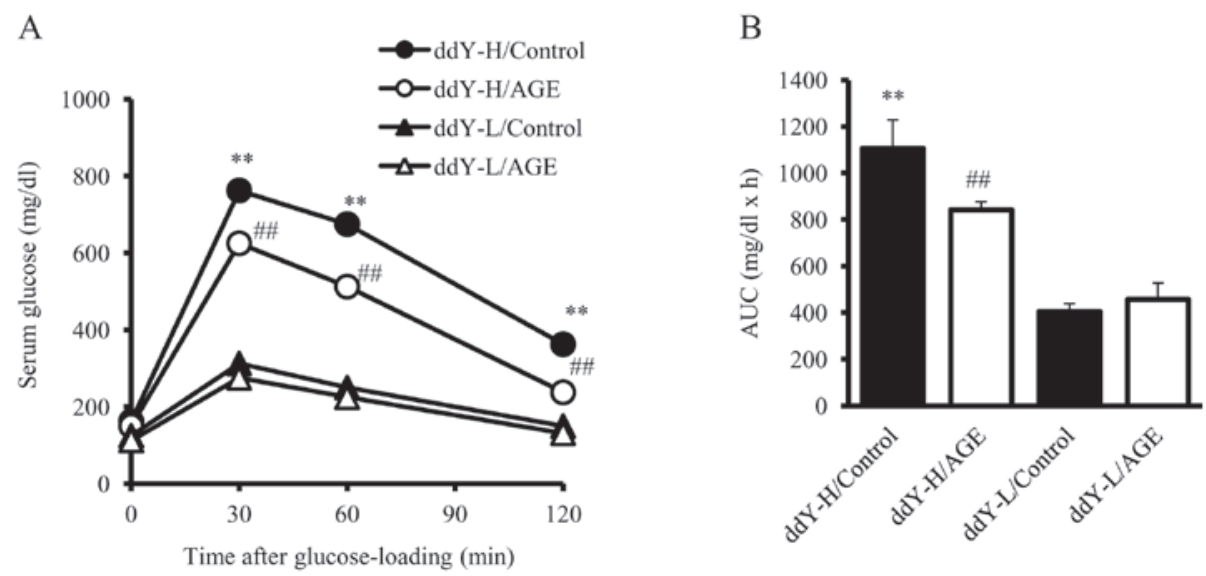

Figure 2. Effect of AGE on oral glucose tolerance test. Mice were fed a standard diet with or without $4 \%$ AGE (w/w) for 7 weeks. Mice were orally administered glucose ( $3 \mathrm{~g} / \mathrm{kg} \mathrm{BW})$ after a 4-h fast, and serum glucose level was measured at the indicated time points. (A and B) Alterations of the serum glucose level and the AUC, respectively. Each symbol and column represents mean for 8 mice. ${ }^{* *} \mathrm{P}<0.01$ vs. ddY-L/Control; ${ }^{* \sharp} \mathrm{P}<0.01$ vs. ddY-H/Control. AGE, aged garlic extract; AUC, area under the curve; BW, body weight.

of the ddY-H mice was significantly greater than that of the ddY-L mice. AGE supplementation also did not influence the BMI in both types of mice (Table I). Thus, although obesity was observed in the ddY-H mice, AGE did not affect dietary intake, body weight and BMI in both types of mice.

Ameliorative effect of AGE on insulin resistance in ddY-H mice. The serum levels of glucose and insulin after AGE-feeding for 7 weeks are presented in Table II. The serum glucose levels in the dd Y-H mice were $44 \%$ higher than those in the ddY-L mice. The administration of AGE significantly reduced the serum glucose levels in the ddY-H mice. Glucose tolerance tests revealed that the glucose tolerance of the ddY-H mice was impaired and that the administration of AGE partly improved the impairment of glucose metabolism (Fig. 2). In addition, the serum insulin levels of the ddY-H mice were significantly increased 5.1-fold compared with those of the ddY-L mice. The elevated serum levels of insulin were partly improved by the administration of AGE to the ddY-H mice (Table II). The insulin sensitivity index and insulin resistance index were evaluated by the serum levels of glucose and insulin measured following a 12-h fast. In the ddY-H mice, the insulin sensitivity index was decreased and the insulin resistance index was significantly increased by comparison with those of the ddY-L mice. The administration of AGE treatment significantly, but partly improved the impairment in the ddY-H mice (Table II). These data suggested that the 


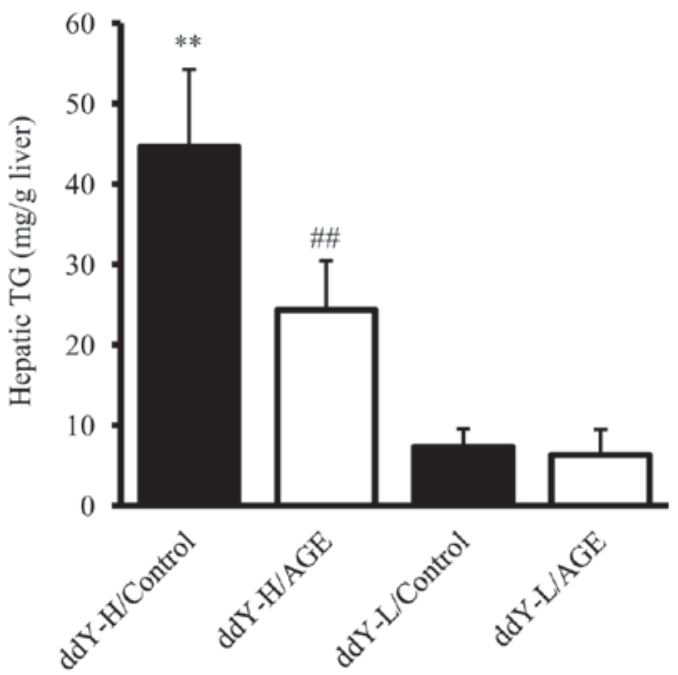

Figure 3. Hepatic TG content in mice. Each column represents the hepatic TG content of mice fed the diets for 7 weeks. Each column represents the means \pm SD for 8 mice. ${ }^{* *} \mathrm{P}<0.01$ vs. ddY-L/Control; ${ }^{\# \#} \mathrm{P}<0.01$ vs. ddY-H/Control. AGE, aged garlic extract; TG, triglycerides.

administration of AGE may ameliorate, at least in part, insulin resistance in ddY-H mice. Since AGE did not influence the serum levels of glucose and insulin (Table II), as well as glucose tolerance (Fig. 2) in the ddY-L mice, the effects of AGE may be limited to abnormal conditions.

Ameliorative effect of AGE on lipid metabolism in ddY-H mice. The effects of AGE on lipid metabolism were examined. The ddY-H mice exhibited a significant elevation in the serum levels of TG by $36 \%$ compared with the ddY-L mice. The administration of AGE had no marked effect on the serum TG levels in either the ddY-H or ddY-L mice (Table II). However, AGE suppressed the massive accumulation of hepatic TG by $47 \%$ in the ddY-H mice (Fig. 3). On the other hand, the administration of AGE did not affect the TG content in the livers of the ddY-L mice (Fig. 3).

The hepatic TG content is regulated via the uptake of fatty acids from the blood, the de novo synthesis of fatty acids, the $\beta$-oxidation of fatty acids and the secretion of lipoproteins into the blood. An impaired regulation may induce a change in the hepatic TG accumulation (45). It has been shown that the absorption of TG from the intestines in ddY-H mice is elevated, but that TG secretion from the liver is not altered (39). In this study, in order to examine the intestinal absorption of fat, olive oil was orally administered and the serum TG level was measured up to $6 \mathrm{~h}$. As shown in Fig. 4A, the serum TG concentration in the ddY-H and ddY-L mice reached the maximum level at $1 \mathrm{~h}$ and then gradually decreased. The TG concentration in the ddY-H mice was 1.7-fold higher compared to that in the ddY-L mice at the peak time and the AUC during the 6-h period in the ddY-H mice was increased 2.3-fold, suggesting the higher absorption of TG from the intestine in ddY-H mice. The administration of AGE significantly reduced the intestinal absorption of fat by $33 \%$ in the ddY-H mice, but not in the ddY-L mice (Fig. 4). These results suggested that AGE suppressed TG accumulation in the livers of ddY-H mice by reducing the intestinal absorption of fat, at least in part.
Intestinal microbiota and the effects of $A G E$ in $d d Y-H$ mice. Recent studies have suggesteded that the intestinal microbiota affects multiple aspects of metabolism in the host and that the alteration of the intestinal flora may contribute to the development of obesity, diabetes, dyslipidemia and NAFLD $(9,10)$ in humans and animal models. Therefore, in this study, the composition of the intestinal microbiota in ddY-H and ddY-L mice was analyzed using a T-RFLP analysis. The compositional ratio of the main microbiota in the feces of mice fed the standard diet at 5,9 and 13 weeks of age is depicted in Fig. 5. No difference was observed between the ratio of microbiota in the ddY-H mice and the ddY-L mice at 5 weeks of age. However, in the ddY-H mice from 9 to 13 weeks of age, the relative presence of Lactobacillales was increased, while that of Bifidobacterium and Prevotella was decreased. The level of Clostridium subcluster XIVa and Bacteroides was not altered. On the other hand, minor microbiota, Clostridium cluster XVIII and Clostridium cluster XI were decreased at 13 weeks, whereas Clostridium cluster IV remained unchanged (Table III). The decrease in the number of Bifidobacterium in feces of ddY-H mice was confirmed by cultivating the feces on BS agar medium under anaerobic conditions and by examination using real-time PCR and the $2^{-\Delta \Delta \mathrm{Cq}}$ method. AGE feeding reversed the altered pattern of microbiota in the feces of ddY-H mice: The relative presence of Lactobacillales was decreased, while that of Bifidobacterium, Clostridium cluster XVIII and Prevotella was increased (Table III). However, no effect of AGE was found in ddY-L mice. Therefore, the composition of microbiota in the feces of the ddY-H mice became similar to that of the ddY-L mice (Table III).

Effect of treatment with antibacterial drugs. Treatment with the antibacterial drugs, ampicillin, vancomycin, neomycin and metronidazole, depletes bacteria in feces (41). In this study, to investigate the possible association of the altered ratio of the gut microbiota to the hepatic TG accumulation and glucose intolerance in ddY-H mice, antibacterial drugs were administered from 6 to 13 weeks of age, and glucose tolerance test and the measurement of the hepatic TG content were carried out. The impaired glucose tolerance of the ddY-H mice was partly improved by treatment with antibacterial drugs (Fig. 6). In addition, the increase in the hepatic TG content in the ddY-H mice was partly decreased by the treatment (Fig. 7). However, treatment did not influence glucose tolerance and the hepatic TG content in the ddY-L mice. These data suggested that glucose intolerance and hepatic TG accumulation in ddY-H mice may be associated with the altered balance of the gut microbiota.

\section{Discussion}

NAFLD has been shown to be closely and bi-directionally associated with insulin resistance and metabolic disease $(7,8)$. In ddY-H mice, metabolic abnormalities, such as obesity, insulin resistance, hyperglycemia, hyperlipidemia and an altered adipokine profile are found at a certain age even when the mice are fed a standard diet without special feeding, such as a high-fat diet $(37,38)$. The hepatic TG level in ddY-H mice increases with the appearance of insulin resistance, and a 
Table III. Effect of AGE on the distribution of microbiota distribution in feces of ddY-H and ddY-L mice. OTU area $(\%)$

\begin{tabular}{|c|c|c|c|c|}
\hline Bacteria & ddY-H/Control & ddY-H/AGE & ddY-L/Control & ddY-L/AGE \\
\hline Bifidobacterium & $3.6 \pm 0.6^{\mathrm{a}}$ & $8.4 \pm 1.5^{\mathrm{b}}$ & $6.0 \pm 1.0$ & $6.7 \pm 1.1$ \\
\hline Lactobacillales & $49.1 \pm 4.0^{\mathrm{a}}$ & $29.5 \pm 2.3^{\mathrm{b}}$ & $22.3 \pm 1.3$ & $20.1 \pm 1.5$ \\
\hline Bacteroides & $24.4 \pm 3.2$ & $21.2 \pm 2.9$ & $30.8 \pm 5.0$ & $27.9 \pm 6.1$ \\
\hline Prevotella & $1.6 \pm 0.2^{\mathrm{a}}$ & $9.5 \pm 0.8^{\mathrm{b}}$ & $5.5 \pm 0.5$ & $7.0 \pm 0.6$ \\
\hline Clostridium cluster IV & $1.0 \pm 0.2$ & $1.7 \pm 0.3$ & $2.9 \pm 0.4$ & $2.8 \pm 0.4$ \\
\hline Clostridium subcluster XIVa & $14.8 \pm 2.1$ & $16.3 \pm 2.2$ & $17.5 \pm 2.1$ & $15.2 \pm 2.1$ \\
\hline Clostridium cluster XI & $1.6 \pm 0.2$ & $3.6 \pm 0.3^{\mathrm{b}}$ & $0.9 \pm 0.2$ & $4.7 \pm 0.6 b$ \\
\hline Clostridium cluster XVIII & $0.2 \pm 0.1^{\mathrm{a}}$ & $4.0 \pm 0.3^{\mathrm{b}}$ & $3.1 \pm 0.3$ & $4.8 \pm 0.5$ \\
\hline Others & $3.7 \pm 0.5^{\mathrm{a}}$ & $5.9 \pm 0.4$ & $11.1 \pm 2.4$ & $10.7 \pm 3.1$ \\
\hline
\end{tabular}

Mice were fed as described in Fig. 1. The extraction of total DNA from feces, polymerase chain reaction and T-RFLP analysis for composition of microbiota in feces were carried out. Length and peak area of T-RFs in electropherograms were divided into 29 OTUs. The OTUs were quantified as percentage of individual OTU area per total OTU area under the curve. Each value represents the means $\pm \mathrm{SD}$ of $6 \mathrm{mice}$. ${ }^{\mathrm{a}} \mathrm{P}<0.01 \mathrm{vs}$. dd Y-L/Control; ${ }^{\mathrm{b}} \mathrm{P}<0.01$ vs. ddY-H/Control. AGE, aged garlic extract; OTU, operational taxonomic unit; T-RF, terminal restriction fragment.

A

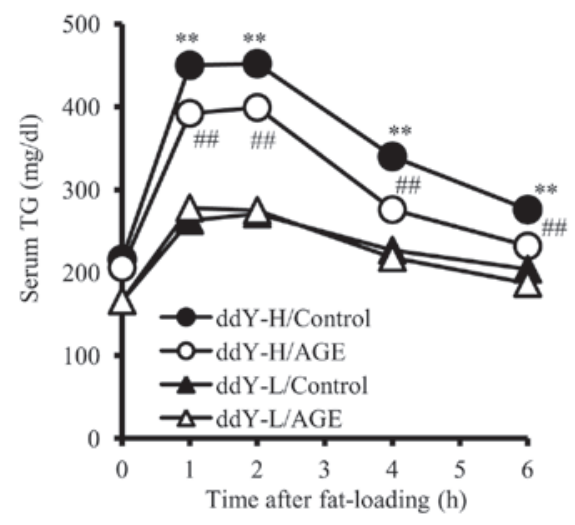

B

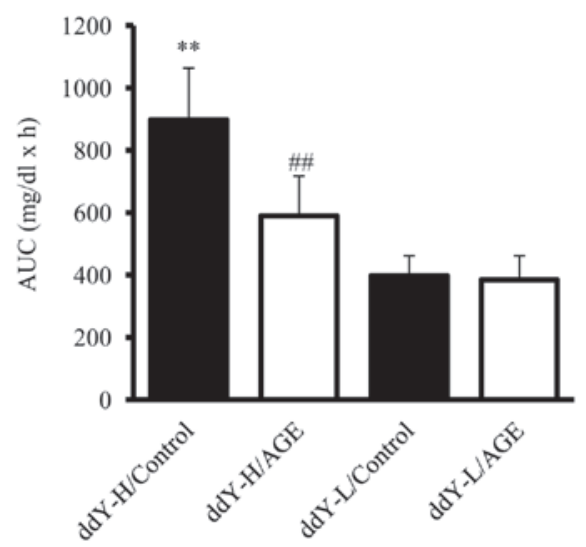

Figure 4. Serum TG concentrations in mice administered olive oil. After being feed the diets for 7 weeks, mice were orally administered olive oil (10 ml/kg BW), and the serum TG levels were determined at the indicated time points. (A) Each symbol represents mean of TG level for 8 mice after olive oil administration. (B) AUC in panel A. Each column represents the means \pm SD for 8 mice. ${ }^{* *} \mathrm{P}<0.01$ vs. ddY-L/Control; ${ }^{\# \#} \mathrm{P}<0.01$ vs. ddY-H/Control. AGE, aged garlic extract; TG, triglycerides; BW, body weight.

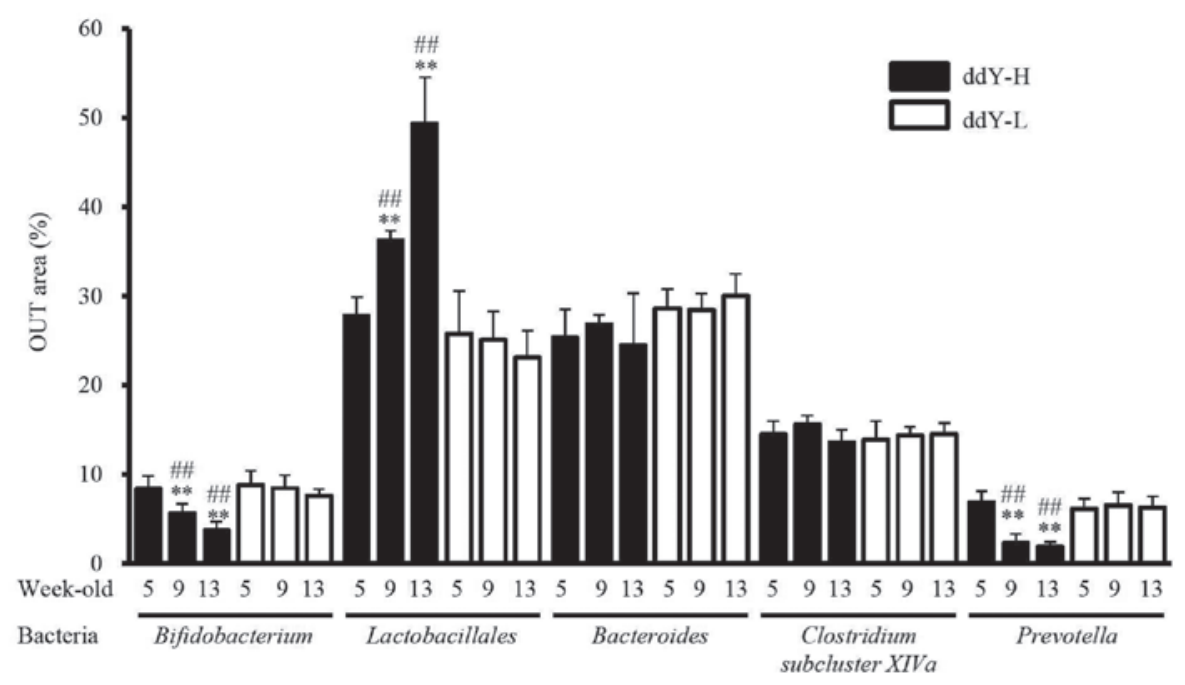

Figure 5. Compositional alteration in the gut microbiota. Main microbiota in feces were analyzed at 5, 9 and 13 weeks of age. Each column represents the means \pm SD for 6 mice. ${ }^{* *} \mathrm{P}<0.01$ vs. ddY-L/Control; ${ }^{\# \#} \mathrm{P}<0.01$ vs. ddY-H/Control. OTU, operational taxonomic unit. 

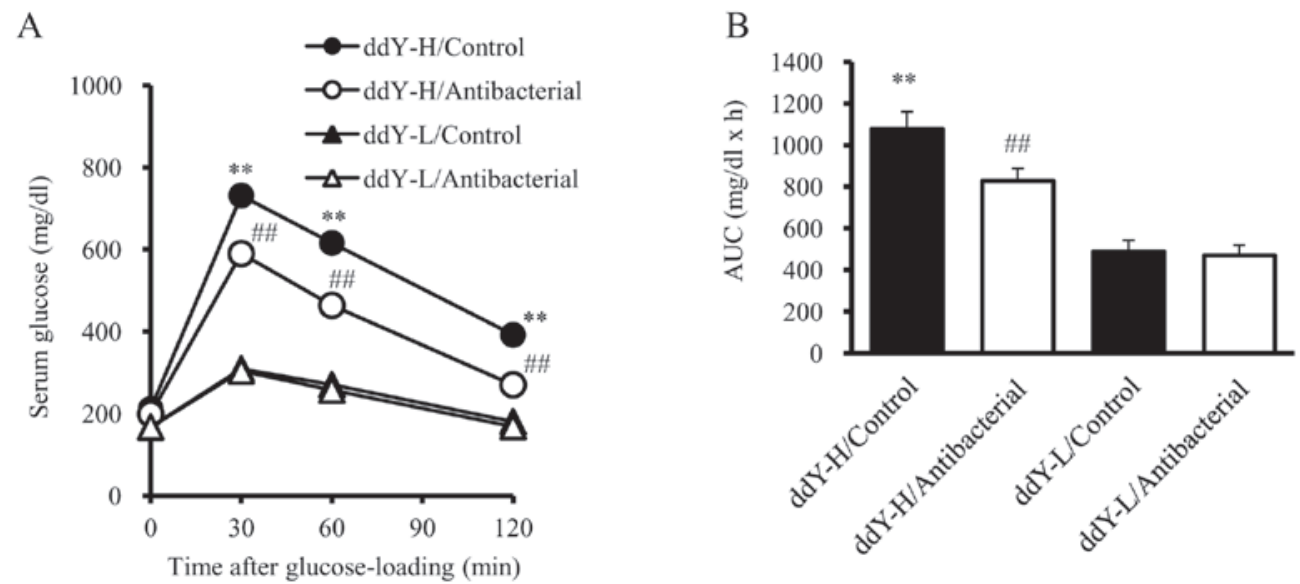

Figure 6. Effect of antibacterial drugs on oral glucose tolerance test. Mice were fed a standard diet and were administered daily antibacterial for 7 weeks. Mice were orally administered glucose (3 g/kg BW) after a $4-\mathrm{h}$ fast, and serum glucose level was measured at the indicated time points. (A and B) Alteration of serum glucose level and the AUC, respectively. Each symbol and column represents the mean for 8 mice. ${ }^{* *} \mathrm{P}<0.01 \mathrm{vs}$. ddY-L/Control; ${ }^{\# \#} \mathrm{P}<0.01$ vs. ddY-H/Control. AUC, area under the curve; BW, body weight.

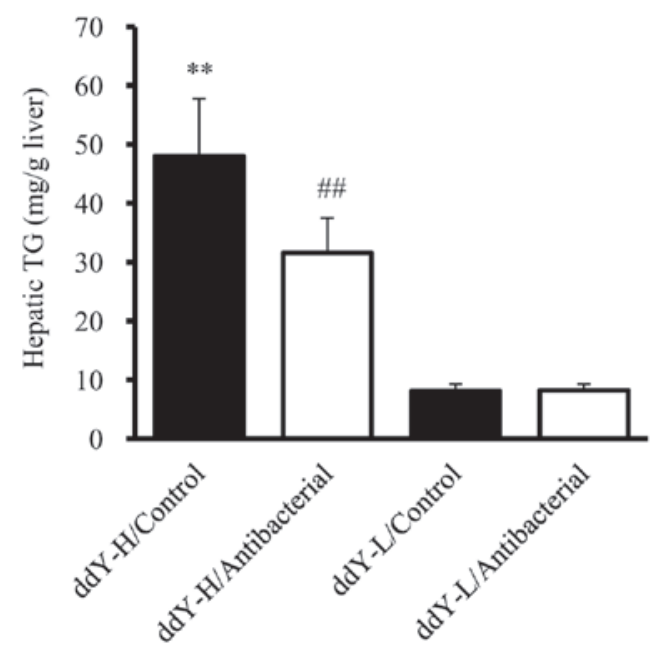

Figure 7. Effect of antibacterial drug on Hepatic TG content in mice. Each column represents hepatic TG content of mice administered antibacterial for 7 weeks. Each column represents the means \pm SD for 8 mice. ${ }^{* *} \mathrm{P}<0.01$ vs. ddY-L/Control; ${ }^{\# \#} \mathrm{P}<0.01$ vs. ddY-H/Control. TG, triglycerides.

marked increase occurs in the liver at 15 and 20 weeks of age (39). However, no histological changes, such as infiltration of inflammatory cells or degeneration, have been observed. In addition, the elevation of serum aspartate aminotransferase and alanine aminotransferase levels has not been found at 15 weeks of age (39). These findings, together with an accumulation of TG in the liver, indicate that NAFLD, but not NASH, is spontaneously induced in ddY-H mice without high-fat diet feeding. Since these abnormalities are observed in patients with NAFLD, the characteristics of ddY-H mice suggest that they may serve a useful model of NAFLD with insulin resistance.

Garlic is known for its medicinal uses as an antibiotic, anti-thrombotic and antineoplastic agent (46). AGE contains various pharmacologically active sulfur-containing compounds that are scarce in cooked or raw garlic (26). AGE has been shown to improve the plasma lipid concentrations and oxidative stress, and inflammatory cytokine levels $(26-27,46,47)$. It has been reported that SAC, a component of AGE, improves
NAFLD in rats with T2D via the regulation of hepatic lipogenesis and glucose metabolism (31). However, the effects of AGE on abnormal fatty acid metabolism in genetic animal models with NAFLD have not been studied to date, at least to the best of our knowledge.

During the progression of diabetes, pancreatic $\beta$ cells adapt their secretory capacity to compensate the elevated glycemia and the peripheral insulin resistance and, as a result, the serum insulin level becomes elevated (48). We have previously shown that insulin resistance in ddY-H mice is induced at 9 weeks of age and develops progressively with age $(37-38,40)$. The age-dependent onset of insulin resistance was closely related to the increase of serum insulin level. The elevation of the serum insulin level was temporally associated with the decrease in mRNA expression and proteins of insulin receptor (IR), IRS-1 and IRS-2 in adipose tissue of ddY-H mice (40). Therefore, together with glucose intolerance, the elevation of serum insulin levels in ddY-H mice indicates an induction of insulin resistance. In this study, the administration of AGE reduced the elevated levels of serum glucose and insulin and also improved the glucose intolerance in ddY-H mice, suggesting that AGE ameliorated insulin resistance in this mouse model, although its effect was not complete.

Fatty acid metabolism in the liver is regulated by a number of factors, including PPAR $\gamma$ (49). PPAR $\gamma$ is a nuclear receptor that activates genes involving in lipid storage and metabolism. It is noteworthy that PPAR $\gamma$ is expressed at an elevated level in the liver of murine models of diabetes or obesity $(50,51)$. We previously demonstrated that treatment with pioglitazone, a PPAR $\gamma$ agonist, exacerbated hepatic the TG deposition in ddY-H mice (39). The mRNA level of PPAR $\gamma$ was markedly elevated in the livers of ddY-H mice, as were the mRNA levels of fatty acid translocase and fat-specific protein 27 , targets of PPAR $\gamma$ (39). These results suggested that the uptake of fatty acids into hepatocytes and the storage of fatty acids as fat droplets contributed to the accumulation of TG in ddY-H mice. AGE may therefore reduce hepatic TG accumulation in ddY-H mice, at least in part, by suppressing the uptake of fat from the intestines and the uptake of fatty acid into hepatocytes. 
The intestinal microbiota forms a complex ecological system that participates, under physiological conditions, in the production of vitamins, the digestion of nutrients and local and general immunity $(52,53)$. It has also been suggested that the intestinal microbiota serves as an important player in the pathophysiology of obesity and its related disorders $(16,17)$. It has been reported that the derangement of the intestinal microbiota occurs in a large percentage of patients with chronic liver disease. In particular, small intestinal bacterial overgrowth is associated with severity in patients with NAFLD (51). Intestinal bacteria may significantly contribute to various liver diseases by activating the innate and adaptive immune responses (52). Dietary habits, lifestyle, age, host genotype and exposure to antibiotics may affect the balance of the intestinal microbiota in humans (52). However, due to the complexity of the microbial community, the functional connection is not yet well understood. The ddY-H mice and ddY-L mice used in this study were bred in the same colony and were maintained by feeding the same diet under the same conditions. In this study, although the difference in bacterial composition in the feces was not found between the ddY-H mice and ddY-L mice at 5 weeks of age, the compositional ratio of some bacteria changed in relation to age in the ddY-H mice. In the ddY-H mice, the relative presence of Lactobacillales was increased, while that of Bifidobacterium, Clostridium cluster XVIII and Prevotella was decreased. The onset of the change was found at the similar age compared with the appearance of insulin resistance and fatty liver. Furthermore, the treatment with antibacterial drugs improved glucose intolerance and hepatic TG accumulation in ddY-H mice. These results suggested the possible association between insulin resistance, fatty liver and the gut microbiota.

The intestinal immune system is known to modulate the gut microbiota and vice versa $(54,55)$. Imbalances of the gut microbiota can trigger several immune disorders through the activity of $\mathrm{T}$ cells that are both near to and distant from the site of their induction (56). Since we maintained ddY-H mice and ddY-L mice under the same conditions, the imbalance in the gut microbiota in ddY-H mice may be possibly related the deterioration of the immune system. The increased mRNA expression of monocyte chemoattractant protein-1 and the infiltration of inflammatory M1 macrophages have been found in the enlarged adipose tissue of ddY-H mice, and inflammation in adipose tissue was considered to be the possible cause inducing insulin resistance (38). The alteration of the gut microbiota composition was temporally associated with the appearance of insulin resistance and NAFLD in ddY-H mice. Recently, evidence has implicated the intestinal immune system as an important contributor to metabolic disease (57). The gut-gut microbiota-liver axis may play an important role in NAFLD (58). Monascus pigments ameliorate the disturbance of lipid metabolism and gut microbiota dysbiosis (59). Metformin, which has been widely used for treatment of T2D, is gaining attention due to its effects on the gut microbiota and the immune system (60). AGE also ameliorates insulin resistance, NAFLD and an imbalance of gut microbiota. Since insulin resistance, NAFLD and microbiota influence each other, AGE may improve the impaired gut-gut microbiota-liver axis in ddY-H mice.

In conclusion, the findings of this study demonstrated AGE ameliorated glucose intolerance and hepatic TG accu- mulation in ddY-H mice, a mouse model of NAFLD. AGE also improved the altered pattern of the intestinal microbiota. These results suggest that the effects of AGE in improving abnormal fat accumulation and insulin resistance may be associated with its effects on the gut microbiota, at least in part.

\section{Acknowledgements}

The authors would like to thank Dr Takami Oka of Wakunaga Pharmaceutical Co., Ltd. for his helpful advice, encouragement and critical reading of the manuscript. The authors would also like to thank Dr Koichi Tamura of Wakunaga Pharmaceutical Co., Ltd. for critically reading the manuscript. The authors are also grateful to Dr Takayoshi Hisada (Techno Suruga Laboratory Co., Ltd.) for providing excellent technical assistance with the microbiota analysis.

\section{Funding}

No funding was received.

\section{Availability of data and materials}

All data generated or analysed during this study are included in this published article.

\section{Authors' contributions}

TM, SM, NM and YK designed the experiments, and TM performed them. TM and YK contributed to the analysis and interpretation of the data. TM wrote the manuscript and SM, NM and YK revised it. All authors have read and approved the final manuscript.

\section{Ethics approval and consent to participate}

Animal care and experiments were performed in accordance with the guidelines for the care and use of laboratory animals of the University of Shizuoka, and animal experiments were reviewed and approved by the Animal and Ethics Review Committee of University of Shizuoka (no. 136004).

\section{Patient consent for publication}

Not applicable.

\section{Competing interests}

The authors declare that they have no competing interests.

\section{References}

1. Brunt EM and Tiniakos DG: Histopathology of nonalcoholic fatty liver disease. World J Gastroenterol 16: 5286-5296, 2010.

2. Angulo P: GI epidemiology: Nonalcoholic fatty liver disease. Aliment Pharmacol Ther 25: 883-889, 2007.

3. Law K and Brunt EM: Nonalcoholic fatty liver disease. Clin Liver Dis 14: 591-604, 2010.

4. Yilmaz Y: Review article: Is non-alcoholic fatty liver disease a spectrum, or are steatosis and non-alcoholic steatohepatitis distinct conditions? Aliment Pharmacol Ther 36: 815-823, 2012. 
5. Samuel VT and Shulman GI: Mechanisms for insulin resistance: Common threads and missing links. Cell 148: 852-871, 2012.

6. Khan R, Bril F, Cusi K and Newsome PN: Modulation of insulin resistance in NAFLD. Hepatology hep.30429, 2018.

7. Lonardo A, Lugari S, Ballestri S, Nascimbeni F, Baldelli E and Maurantonio M: A round trip from nonalcoholic fatty liver disease to diabetes: Molecular targets to the rescue? Acta Diabetol 56:385-396, 2018.

8. Kim JY, Park KJ, Hwang JY, Kim GH, Lee D, Lee YJ, Song $\mathrm{EH}$, Yoo MG, Kim BJ, Suh YH, et al: Activating transcription factor 3 is a target molecule linking hepatic steatosis to impaired glucose homeostasis. J Hepatol 67: 349-359, 2017.

9. Gérard P: Gut microbiota and obesity. Cell Mol Life Sci 73: 147-162, 2016.

10. Saad MJ, Santos A and Prada PO: Linking gut microbiota and inflammation to obesity and insulin resistance. Physiology (Bethesda) 31: 283-293, 2016.

11. Chi Y, Lin Y, Lu Y, Huang Q, Ye G and Dong S: Gut microbiota dysbiosis correlates with a low-dose PCB126-induced dyslipidemia and non-alcoholic fatty liver disease. Sci Total Environ 653: 274-282, 2019.

12. Safari $Z$ and Gerard P: The links between the gut microbiome and non-alcoholic fatty liver disease (NAFLD). Cell Mol Life Sci 76: 1541-1558, 2019

13. Ma J, Zhou Q and Li H: Gut microbiota and nonalcoholic fatty liver disease: Insights on mechanisms and therapy. Nutrients 9 : 1127-1147, 2017.

14. Cho MS, Kim SY, Suk KT and Kim BY: Modulation of gut microbiome in nonalcoholic fatty liver disease: Pro-, pre-, synand antibiotics. J Microbiol 56: 855-867, 2018.

15. Sedighi M, Razavi S, Navab-Moghadam F, Khamseh ME, Alaei-Shahmiri F, Mehrtash A and Amirmozafari N: Comparison of gut microbiota in adult patients with type 2 diabetes and healthy individuals. Microb Pathog 111: 362-369, 2017.

16. Bagarolli RA, Tobar N, Oliveira AG, Araújo TG, Carvalho BM Rocha GZ, Vecina JF, Calisto K, Guadagnini D, Prada PO, et al: Probiotics modulate gut microbiota and improve insulin sensitivity in DIO mice. J Nutr Biochem 50: 16-25, 2017.

17. Cani PD, Bibiloni R, Knauf C, Waget A, Neyrinck AM, Delzenne $\mathrm{NM}$ and Burcelin R: Changes in gut microbiota control metabolic endotoxemia-induced inflammation in high-fat diet-induced obesity and diabetes in mice. Diabetes 57: 1470-1481, 2008

18. Zhou Y, Zheng T, Chen H, Li Y, Huang H, Chen W, Du Y, He J, Li Y, Cao J, et al: Microbial intervention as a novel target in treatment of non-alcoholic fatty liver disease progression. Cell Physiol Biochem 51: 2123-2135, 2018.

19. Perumpail BJ, Li AA, John N, Sallam S, Shah ND, Kwong W, Cholankeril G, Kim D and Ahmed A: The therapeutic implications of the gut microbiome and probiotics in patients with NAFLD. Diseases 7: 1-12, 2019.

20. Chen S, Shen X, Cheng S, Li P, Du J, Chang Y and Meng H: Evaluation of garlic cultivars for polyphenolic content and antioxidant properties. PLoS One 8: e79730, 2013.

21. Kim MJ, Yoo YC, Kim HJ, Shin SK, Sohn EJ, Min AY, Sung NY and Kim MR: aged black garlic exerts anti-inflammatory effects by decreasing no and proinflammatory cytokine production with less cytoxicity in LPS-stimulated raw 264.7 macrophages and LPS-induced septicemia mice. J Med Food 17: 1057-1063, 2014.

22. Jang HJ, Lee HJ, Yoon DK, Ji DS, Kim JH and Lee CH: Antioxidant and antimicrobial activities of fresh garlic and aged garlic by-products extracted with different solvents. Food Sci Biotechnol 27: 219-225, 2017

23. Bordia T, Mohammed N, Thomson M and Ali M: An evaluation of garlic and onion as antithrombotic agents. Prostaglandins Leukot Essent Fatty Acids 54: 183-186, 1996.

24. Alpers DH: Garlic and its potential for prevention of colorectal cancer and other conditions. Curr Opin Gastroenterol 25: 116-121, 2009

25. Miki S, Inokuma KI, Takashima M, Nishida M, Sasaki Y, Ushijima M, Suzuki JI and Morihara N: Aged garlic extract suppresses the increase of plasma glycated albumin level and enhances the AMP-activated protein kinase in adipose tissue in TSOD mice. Mol Nutr Food Res 61: 5-11, 2017.

26. Amagase $\mathrm{H}$, Petesch BL, Matsuura $\mathrm{H}$, Kasuga $\mathrm{S}$ and Itakura $\mathrm{Y}$ : Intake of garlic and its bioactive components. J Nutr 131: 955S-962S, 2001.

27. Yeh YY and Liu L: Cholesterol-lowering effect of garlic extracts and organosulfur compounds: Human and animal studies. J Nutr 131: 989S-993S, 2001
28. Wang BH, Zuzel KA, Rahman K and Billington D: Treatment with aged garlic extract protects against bromobenzene toxicity to precision cut rat liver slices. Toxicology 132: 215-225, 1999.

29. Sumioka I, Matsura T and Yamada K: Therapeutic effect of S-allylmercaptocysteine on acetaminophen-induced liver injury in mice. Eur J Pharmacol 433: 177-185, 2001.

30. Kodai S, Takemura S, Minamiyama Y, Hai S, Yamamoto S, Kubo S, Yoshida Y, Niki E, Okada S, Hirohashi K and Suehiro S: S-allyl cysteine prevents $\mathrm{CCl}(4)$-induced acute liver injury in rats. Free Radic Res 41: 489-497, 2007.

31. Takemura S, Minamiyama Y, Kodai S, Shinkawa H, Tsukioka T, Okada S, Azuma $\mathrm{H}$ and Kubo S: S-Allyl cysteine improves nonalcoholic fatty liver disease in type 2 diabetes Otsuka Long-Evans Tokushima Fatty rats via regulation of hepatic lipogenesis and glucose metabolism. J Clin Biochem Nutr 53: 94-101, 2013.

32. Ried K, Travica N and Sali A: The effect of Kyolic aged garlic extract on gut microbiota, inflammation, and cardiovascular markers in hypertensives: The GarGIC Trial. Front Nutr 5: 122-136, 2018.

33. Lieber CS, Leo MA, Mak KM, Xu Y, Cao Q, Ren $C$, Ponomarenko A and DeCarli LM: Model of nonalcoholic steatohepatitis. Am J Clin Nutr 79: 502-509, 2004.

34. Brix AE, Elgavish A, Nagy TR, Gower BA, Rhead WJ and Wood PA: Evaluation of liver fatty acid oxidation in the leptin-deficient obese mouse. Mol Genet Metab 75: 219-226, 2002

35. Wortham M, He L, Gyamfi M, Copple BL and Wan YJ: The transition from fatty liver to NASH associates with SAMe depletion in $\mathrm{db} / \mathrm{db}$ mice fed a methionine choline-deficient diet. Dig Dis Sci 53: 2761-2774, 2008

36. Okumura K, Ikejima K, Kon K, Abe W, Yamashina S, Enomoto N, Takei Y and Sato N: Exacerbation of dietary steatohepatitis and fibrosis in obese, diabetic KK-A(y) mice. Hepatol Res 36: 217-228, 2006

37. Noge I, Kagawa Y and Maeda T: A new diabetic mouse model derived from the ddY strain. Biol Pharm Bull 33: 988-992, 2010

38. Maeda T, Noge I and Kagawa Y: Infiltration of M1 macrophages into adipose tissue of ddY-H mice preceding spontaneous appearances of insulin resistance. Biol Pharm Bull 36: 825-832, 2013.

39. Satoh H, Ide N, Kagawa Y and Maeda T: Hepatic steatosis with relation to increased expression of peroxisome proliferator-activated receptor- $\gamma$ in insulin resistant mice. Biol Pharm Bull 36: 616-623, 2013.

40. Ide N, Arisawa K, Ogura K, Kagawa Y and Maeda T: Age-dependent onset of insulin resistance in insulin-resistant mice. Biol Pharm Bull 38: 1925-1934, 2015.

41. Rakoff-Nahoum S, Paglino J, Eslami-Varzaneh F, Edberg S and Medzhitov R: Recognition of commensal microflora by toll-like receptors is required for intestinal homeostasis. Cell 118: 229-241, 2004

42. Nagashima K, Hisada T, Sato M and Mochizuki J: Application of new primer-enzyme combinations to terminal restriction fragment length polymorphism profiling of bacterial populations in human feces. Appl Environ Microbiol 69: 1251-1262, 2003.

43. Nagashima K, Mochizuki J, Hisada T, Suzuki S and Shimomura K: Phylogenetic analysis of 16s ribosomal RNA gene sequences from human fecal microbiota and improved utility of terminal restriction fragment length polymorphism profiring. Biosci Microflora 25: 99-107, 2006.

44. Livak KJ and Schmittgen TD: Analysis of relative gene expression data using real-time quantitative PCR and the $2^{(-\Delta \Delta C(\mathrm{~T}))}$ method. Methods 25: 402-408, 2001

45. Ferramosca A and Zara V: Modulation of hepatic steatosis by dietary fatty acids. World J Gastroenterol 20: 1746-1755, 2014.

46. Ried K, Travica N and Sali A: The effect of aged garlic extract on blood pressure and other cardiovascular risk factors in uncontrolled hypertensives: The AGE at Heart trial. Integr Blood Press Control 9: 9-21, 2016.

47. Rana SV, Pal R, Vaiphei K, Sharma SK and Ola RP: Garlic in health and disease. Nutr Res Rev 24: 60-71, 2011.

48. Rabhi N, Salas E, Froguel P and Annicotte JS: Role of the unfolded protein response in $\beta$ cell compensation and failure during diabetes. J Diabetes Res 2014: 795171, 2014.

49. Memon RA, Tecott LH, Nonogaki K, Beigneux A, Moser AH, Grunfeld $C$ and Feingold KR: Up-regulation of peroxisome proliferator-activated receptors (PPAR-alpha) and PPAR-gamma messenger ribonucleic acid expression in the liver in murine obesity: Troglitazone induces expression of PPAR-gamma-responsive adipose tissue-specific genes in the liver of obese diabetic mice. Endocrinology 141: 4021-4031, 2000. 
50. Rahimian R, Masih-Khan E, Lo M, van Breemen $\mathrm{C}$ McManus BM and Dubé GP: Hepatic over-expression of peroxisome proliferator activated receptor gamma2 in the ob/ob mouse model of non-insulin dependent diabetes mellitus. Mol Cell Biochem 224: 29-37, 2001.

51. Nagata K, Suzuki H and Sakaguchi S: Common pathogenic mechanism in development progression of liver injury caused by non-alcoholic or alcoholic steatohepatitis. J Toxicol Sci 32: 453-468, 2007.

52. Compare D, Coccoli P, Rocco A, Nardone OM, De Maria S, Cartenì $\mathrm{M}$ and Nardone G: Gut--liver axis: The impact of gut microbiota on non alcoholic fatty liver disease. Nutr Metab Cardiovasc Dis 22: 471-476, 2012.

53. O'Hara AM and Shanahan F: The gut flora as a forgotten organ. EMBO Rep 7: 688-693, 2006.

54. Burcelin R: Gut microbiota and immune crosstalk in metabolic disease. Biol Aujourdhui 211: 1-18, 2017.

55. Logan AC, Jacka FN and Prescott SL: Immune-microbiota interactions: dysbiosis as a global health issue, Curr Allergy Asthma Rep 16: 13, 2016.
56. Honda $\mathrm{K}$ and Littman DR: The microbiota in adaptive immune homeostasis and disease. Nature 535: 75-84, 2016.

57. Winer DA, Luck H, Tsai S and Winer S: The intestinal immune system in obesity and insulin resistance. Cell Metab 23: 413-426, 2016.

58. Suk KT and Kim DJ: Gut microbiota: Novel therapeutic target for nonalcoholic fatty liver disease. Expert Rev Gastroenterol Hepatol 13: 193-204, 2019.

59. Zhou W, Guo R, Guo W, Hong J, Li L, Ni L, Sun J, Liu B, Rao P and Lv X: Monascus yellow, red and orange pigments from red yeast rice ameliorate lipid metabolic disorders and gut microbiota dysbiosis in Wistar rats fed on a high-fat diet. Food Funct 10: 1073-1084, 2019.

60. Pollak M: The effects of metformin on gut microbiota and the immune system as research frontiers. Diabetologia 60 1662-1667, 2017 